\title{
DISCRETE MATHEMATICS
}

Proof Techniques and Mathematical Structures 
This page is intentionally left blank 


\section{DISCRETE MATHEMATICS}

\section{Proof Techniques and Mathematical Structures}

\section{R C Penner}

Departments of Mathematics and Physics

University of Southern California 


\title{
Published by
}

World Scientific Publishing Co. Pte. Ltd.

P O Box 128, Farrer Road, Singapore 912805

USA office: Suite 1B, 1060 Main Street, River Edge, NJ 07661

UK office: 57 Shelton Street, Covent Garden, London WC2H 9HE

\author{
Library of Congress Cataloging-in-Publication Data \\ Penner, R. C., 1956 \\ Discrete mathematics : proof techniques and mathematical structures / R. C. Penner. \\ p. $\mathbf{c m}$. \\ Includes bibliographical references and index. \\ ISBN 9810240880 \\ 1. Mathematics. 2. Computer science--Mathematics. I. Title. \\ QA39.2.P44 1999 \\ $510.21--\mathrm{dc} 21$ \\ 99-044793 \\ CIP
}

\section{British Library Cataloguing-in-Publication Data}

A catalogue record for this book is available from the British Library.

First published 1999

Reprinted 2001

Copyright $\odot 1999$ by World Scientific Publishing Co. Pte. Ltd.

All rights reserved. This book, or parts thereof, may not be reproduced in any form or by any means, electronic or mechanical, including photocopying, recording or any information storage and retrieval system now known or to be invented, without written permission from the Publisher.

For photocopying of material in this volume, please pay a copying fee through the Copyright Clearance Center, Inc., 222 Rosewood Drive, Danvers, MA 01923, USA. In this case permission to photocopy is not required from the publisher.

Printed in Singapore by Uto-Print 
Dedicated to my mother, Beverly Preston Penner, a teacher

Written for my children, Kirby and Michael 
This page is intentionally left blank 


\section{Contents}

General Preface .. . . . . . . . . . . . . . . . . xi

Preface for Instructors . . . . . . . . . . . . . . . . . . . . xii

\section{PART 1}

Chapter 1 Proof Techniques 1

Part A Elements of Logic and Induction 2

A.1 Definitions . . . . . . . . . . . . . . . . . . . 2

A.2 Propositions and Predicates . . . . . . . . . . . . . . . . . 3

A.3 Conjunction and Negation . . . . . . . . . . . . . . . . . . 7

A.4 Implication . . . . . . . . . . . . . . . . . . . . . . . . . 9

A.5 Proofs by Induction for Natural Numbers . . . . . . . . . . . 12

Part B Methods of Proof $\quad 24$

B.1 Chains of Implications . . . . . . . . . . . . . . . . . . . . 24

B.2 Proof by Contradiction . . . . . . . . . . . . . . . . . . . . 32

B.3 Instantiation . . . . . . . . . . . . . . . . . . . . . 38

B.4 Constructive and Non-Constructive Proofs . . . . . . . . . . . 40

B.5 Disproofs . . . . . . . . . . . . . . . . . . . . . . . . . . 43

Chapter 2 Predicate Calculus . . . . . . . . . . . . . . . . . . .45

2.1 Logical Operators . . . . . . . . . . . . . . . . . 46

2.2 Propositional Forms . . . . . . . . . . . . . . . . . . . 48

2.3 Parentheses . . . . . . . . . . . . . . . . . . . . 50

2.4 Standard Logical Identities . . . . . . . . . . . . . . . . . 53

2.5 Standard Rules of Inference . . . . . . . . . . . . . . . . . . 55

(*)2.6 Aristotelean Logic . . . . . . . . . . . . . . . . . . . . . . 57

2.7 Logical Equivalence . . . . . . . . . . . . . . . . . . . . . 62

(*)2.8 Commutativity and Associativity . . . . . . . . . . . . 68

2.9 Proving Propositional Forms . . . . . . . . . . . . . . . . . 72

2.10 Predicate Forms and Quantification . . . . . . . . . . . . . 74

2.11 Standard Valid Predicate Forms . . . . . . . . . . . . . . . 80

2.12 Proving Predicate Forms . . . . . . . . . . . . . . . . . . 83

2.13 Disproofs . . . . . . . . . . . . . . . . . . 86

Chapter 3 Set Theory . . . . . . . . . . . . . . . . . . . . . . 93

3.1 Axioms and the Primitives of Set Theory . . . . . . . . . . . . 94 
3.2 The Basics of Set Theory . . . . . . . . . . . . . . . . . 98

(*)3.3 ZFC Set Theory . . . . . . . . . . . . . . . . . . . . . . 107

3.4 Binary Operations on Sets . . . . . . . . . . . . . . . . 114

3.5 Cartesian Products . . . . . . . . . . . . . . . . . . . . 122

3.6 Absolute Complements and DeMorgan's Laws . . . . . . . . . 127

(*)3.7 The Set of Non-Negative Integers . . . . . . . . . . . . . . 134

3.8 Inductive Definitions . . . . . . . . . . . . . . . . . . 141

3.9 Sets of Numbers . . . . . . . . . . . . . . . . . . . 150

$\begin{array}{ll}\text { Chapter } 4 \text { Elementary Number Theory } & 151\end{array}$

4.1 Common Multiples . . . . . . . . . . . . . . . . . . . . . 152

4.2 The Division Algorithm . . . . . . . . . . . . . . . . . . 154

4.3 Common Divisors . . . . . . . . . . . . . . . . . . . . . 156

4.4 Relatively Prime Pairs . . . . . . . . . . . . . . . . . 157

4.5 Linear Diophantine Equations . . . . . . . . . . . . . . . . 159

4.6 The Fundamental Theorem of Arithmetic . . . . . . . . . . . 164

4.7 The Euclidean Algorithm . . . . . . . . . . . . . . . . . . 168

(*)4.8 Continued Fractions . . . . . . . . . . . . . . . . . . . . 172

$\begin{array}{lr}\text { Chapter } 5 \text { Relations } & 179\end{array}$

$5.1 \mathrm{~N}$-ary Relations . . . . . . . . . . . . . . . . 180

5.2 Binary Relations and Digraphs . . . . . . . . . . . . . . . 184

5.3 Properties of Relations . . . . . . . . . . . . . . . . . . . 189

5.4 Set-Theoretic Operations . . . . . . . . . . . . . . . . . . 194

5.5 Inversion . . . . . . . . . . . . . . . . . . . 196

5.6 Composition . . . . . . . . . . . . . . . . . . . . . . 198

(*)5.7 Iterations . . . . . . . . . . . . . . . . . . . . . . 203

5.8 Posets . . . . . . . . . . . . . . . . . . . 208

5.9 Linear and Well Orders . . . . . . . . . . . . . . . . . . 214

(*)5.10 Bounds in Posets . . . . . . . . . . . . . . . . . . . . . 222

(*)5.11 Axiom of Choice Revisited . . . . . . . . . . . . . . . . . 225

5.12 Equivalence Relations . . . . . . . . . . . . . . . 227

5.13 Partitions . . . . . . . . . . . . . . . . . . . . . 234

(*)5.14 Closure Operations . . . . . . . . . . . . . . . . . . . . 241

(*)5.15 Meets and Joins of Partitions . . . . . . . . . . . . . . . . 249

(*)5.16 Lattices . . . . . . . . . . . . . . . . . . . . . 252

Chapter 6 Functions $\quad 259$

6.1 Definitions and Examples . . . . . . . . . . . . . . 260

6.2 Composition . . . . . . . . . . . . . . . . . . . . . . . 269

6.3 Restriction and Extension . . . . . . . . . . . . . 275

6.4 Injectivity, Surjectivity, and Bijectivity . . . . . . . . . . 278

6.5 Inverses . . . . . . . . . . . . . . . . . . . . . 285

6.6 Images and Pre-Images . . . . . . . . . . . . . . . . . . 292 
(*)6.7 Elements of Dynamical Systems . . . . . . . . . . . . . 301

(*)6.8 Permutations . . . . . . . . . . . . . . . . . . . . . . . 307

\section{PART 2}

Chapter 7 Cardinality $\quad 313$

7.1 Finite Sets . . . . . . . . . . . . . . . . . . . . . . . . 314

7.2 Infinite Sets . . . . . . . . . . . . . . . . . . . . . . . . 319

7.3 Countable Sets . . . . . . . . . . . . . . . . . . . . 322

7.4 Uncountable Sets . . . . . . . . . . . . . . . . . . . . . 328

7.5 Comparing Cardinalities . . . . . . . . . . . . . . . . 332

$\begin{array}{ll}\text { Chapter } 8 \text { Elements of Combinatorics } & 341\end{array}$

8.1 Finite Cardinalities . . . . . . . . . . . . . . . . . . . . 342

8.2 Combinatorial Proofs . . . . . . . . . . . . . . . . . . . . 345

8.3 Recursively Defined Sequences . . . . . . . . . . . . . . . . 353

8.4 Inductive Proofs and Solving Recursions . . . . . . . . . . . 361

(†)8.5 Characteristic Equations . . . . . . . . . . . . . . . . . . 368

(†)8.6 Generating Functions . . . . . . . . . . . . . . . . . . . 374

Chapter 9 Languages and Finite State Automata 383

9.1 Alphabets, Words, and Concatenation . . . . . . . . . . . . 384

9.2 Languages . . . . . . . . . . . . . . . . . . . . . . . . 388

9.3 Regular Expressions . . . . . . . . . . . . . . . . . . . . 396

9.4 Finite State Automata . . . . . . . . . . . . . . . . . . . 399

(†)9.5 Kleene's Theorem . . . . . . . . . . . . . . . . . . . . . 408

(†)9.6 Semigroups and Monoids . . . . . . . . . . . . . . . . . 417

Chapter 10 Graphs $\quad \mathbf{4 2 5}$

10.1 Definitions and Examples . . . . . . . . . . . . . . 426

10.2 Trees . . . . . . . . . . . . . . . . . . . . . . . . . . . 432

(†)10.3 Hamiltonian and Eulerian Paths . . . . . . . . . . . . . . . 438

(†) 10.4 Planarity . . . . . . . . . . . . . . . . . . . . . . . . . 441

(†)10.5 Connectivity, Matching, and Coloring . . . . . . . . . 446

Suggestions for Further Reading 455

$\begin{array}{ll}\text { Index of Notation } & 456\end{array}$

$\begin{array}{ll}\text { Index } & \mathbf{4 5 9}\end{array}$ 
This page is intentionally left blank 


\section{General Preface}

I am neither joking nor exaggerating when I say that there are no real prerequisites for reading this book: Chapter 1 begins tabula rasa, that is, with a clean slate, except for a passing familiarity with basic mathematical results from high school such as the quadratic formula, Pythagoras' theorem, and so on (and these results are actually used only for motivation in the early chapters).

At the same time that the pre-requisites are minimal, the goal is no less than to teach the "beginning" math student how to discover, prove, and check mathematics as well as how to write down proofs; this involves not only the discovery and exposition of proofs but also learning the basic constructions and vocabulary of mathematics. Often at American colleges and universities, this is the purview of the first post-calculus mathematics course often for computer science as well as math majors (and sometimes it is hurriedly treated as a first topic in a junior- or senior-level course on modern algebra). On the other hand, this volume does not depend at all upon differential calculus (the only exception being that certain exercises, which are independent of the main text, sometimes do depend upon calculus). The book could quite well be studied much earlier though it does assume a modicum of that elusive attribute "intellectual maturity" on the part of the reader. It is also a suitable text for an upper-division college course with several different possible emphases.

This text has two parts, and Part 1 is the "core" of a one-semester course upon which this volume is based which covers proofs and the foundations of discrete mathematics. Part 2 consists of several independent chapters which apply the constructions and techniques of Part 1 to a more advanced study of various topics in pure math, applied math, and computer science. Part 2 is certainly more advanced and intrinsically interesting than Part 1 , and the reader might rightly regard studying Part 1 as the price to be paid in order that Part 2 be accessible.

At the same time that there have been the two goals of explaining proofs and teaching the basic constructions of mathematics, there have likewise been two guiding virtues: truth and beauty. By this I mean that there have been no pedagogical short-cuts or lies (for how can one teach intellectual rigor without being explicit and honest about the assumptions?), and I have at each stage strived to go beyond the standard material and naively present more advanced and "beautiful" mathematics than is usually covered in a course at this level in both Parts 1 and 2. This deeper and more interesting material is often covered in optional sections, which can be judiciously included to tailor a suitable course for a particular audience. This has actually been another guiding principle: to keep the text as flexible as possible by allowing many different courses based upon different paths through the volume. Thus, this text surely contains more material than any particular syllabus based upon it, I believe this is appropriate and desirable, and the reader is heartily encouraged to "poke around" in this volume beyond any assigned reading.

There are two acknowledgements I want to make here, one from my mathematical childhood (by which I mean my undergraduate days) and one more recent. For the former, I thank my long-ago teachers Anil Nerode and Michael Morley for 
instilling my abiding love for the foundations of mathematics, and I can only hope that this volume might inspire a student as Professor Nerode's notes on related material once inspired me on to a career in research mathematics.

More recently, I have taught the course Proof Techniques and Mathematical Structures at the University of Southern California often over the last decade, and I thank the many students who have helped in the evolution of this manuscript as well as various colleagues at USC, especially the late Dennis Estes, for valuable input. Let me finally acknowledge that work on this volume has been partially supported by the National Science Foundation.

\section{Preface for Instructors}

As mentioned above, Part 1 more or less covers the "core" of a one-semester course on the foundations of math. (As an aside, we note that this book has also been used successfully for independent reading both by strong undergraduates and as a remedial for graduate students.) This foundational material includes predicate calculus, set theory, elementary number theory, relations, and functions. At the college level, there has typically been sufficient time in this course for a "final topic" following this core material, which can either be culled from the independent chapters of Part 2 or else treated concurrently as included optional sub-topics from Part 1. These optional sections in Part 1 are marked with an asterisk $\left({ }^{*}\right)$, and they may be skipped entirely without affecting the continuity of core sections. (The only logical dependence is that later optional sections may depend upon earlier ones but only within the same chapter.) There are many sensible paths through this volume, therefore, by judiciously including various consecutive optional sections within a chapter from Part 1, or by omitting any or all such optional sections and instead moving on to more advanced topics in Part 2.

The various chapters in Part 2 are entirely independent, and each is organized so that the sections become increasingly more sophisticated; we have tried to include numerous intellectually satisfying places within each chapter in Part 2 at which a course might end. The final sections of a chapter in Part 2 marked with a dagger $(\dagger)$ are entirely independent from one another, and each treats a major principle or proves a major theorem. Thus, a chapter or various fractions of various chapters from Part 2 could reasonably be included in a syllabus.

Though the topics and general flow of Part 1 can be gleaned by perusing the Table of Contents, we next serially discuss aspects of each chapter with an emphasis on the optional sections.

Chapter 1 contains Parts A and B, and Part A is a short and gentle introduction to induction and to basic concepts in logic. Part B discusses methods of prool giving numerous classical examples. We have typically lectured only selectively and briefly on the material in Part B and assigned the bulk of this material tc independent reading in the first week or so of class. The idea is that with just a minimal background, essentially all of the standard methods of proof can be palatably described and presented. At the same time, comprehensively present- 
ing the predicate calculus before discussing methods of proof tends to overwhelm the student, who may lose sight of the forest for the trees. In particular, it is useful and practical to present a first view of induction in this somewhat informal setting. We have included general exercises on proof techniques (though we usually do not assign such problems ourselves for reasons mentioned parenthetically above).

Chapter 2 is dedicated to a complete treatment of the predicate calculus with an emphasis on the nuts and bolts of calculational techniques. The section $\S 2.6$ on Aristotelean logic is optional because it is too elementary for many students (and gives practice in "translating" between English and the predicate calculus for whomever it may be necessary) and is a sensible reading assignment. $§ 2.8$ on commutativity and associativity is "extremely optional" and should almost certainly be relegated to reading or ignored altogether; it is included only for (the perspicacious student who may demand) completeness.

Chapter 3 treats set theory, and the optional $\S 3.3$ on ZFC gives a complete treatment of Zermelo-Fraenkel set theory and the Axiom of Choice; actually, most of the axioms of ZFC are presented in the (non-optional) previous section since they are required for our careful treatment of sets. Furthermore, ZFC gives a nice application of the predicate calculus as well as a nice example of an axiomatic mathematical definition. (In some texts at this level, the real numbers are given as the prototypical example of an axiomatic definition, and we believe this is overwhelming for the student; furthermore, a careful definition of the reals is the purview of a first course in analysis or topology in our opinion. The real numbers are employed in this book just in order to give illustrative examples of various constructions, we require essentially no formal properties of them, and hence the term "discrete mathematics" in our title.) We also allow ourselves a certain license in discussing philosophical aspects of these foundational issues. The other optional section $\S 3.7$ gives a complete and careful definition of the natural numbers and includes a survey of recursion and arithmetic (again for that perspicacious student).

In Chapter 4, we break from an exposition of the foundations of mathematics to discuss elementary number theory. The point of this chapter is to convince the reader of the efficacy and power of what has come before in the context of a topic which the reader presumably "mastered" in elementary school. The chapter requires roughly two weeks of lectures, begins with the basics, and ends with a brief discussion of more advanced material, namely, the Euclidean algorithm and continued fractions. In fact, this entire chapter is "optional" (and later chapters are essentially independent of it) but highly recommended to prevent the student from being overwhelmed by the definitions and formalism and losing sight of the goals of the core course.

Chapter 5 returns to the systematic development of foundations with a complete treatment of relations, and it is truly the "heart" of Part 1. Optional sections include treatments of iterations, closure operations, meets and joins of partitions, and the basics of lattice theory. An upper-division college course could begin with Chapters 5 and 6 and then quickly move on to selected topics in Part 2. 
We turn finally to a discussion of functions.in Chapter 6 covering more material than is customary including restriction and extension of domain and range, algebras of real-valued functions, and the usual "categorical" material of inclusion, projection, quotient maps, and so on. There is an optional section on dynamical systems with applications in the next optional section to the study of the symmetric groups. We believe these are essentially ideal additional topics since the treatment of permutations and dynamics here helps to instill a proper intuition regarding functions.

This completes our serial discussion of Part 1. Highlights from the optional sections of Part 2 include treatments of generating functions and characteristic equations (where we proceed by "guessing" and then proving the main theorem) in Chapter 8. Kleene's Theorem and a treatment of semi-groups and monoids are given in Chapter 9 (and a further discussion of algebraic structures is the purview, we believe, of a first course in modern algebra), and Chapter 10 contains some of the great combinatorics results of the 1920-30's of Hall and Menger as well as a glimpse of Ramsey theory, the chromatic polynomial of a graph, and an explicit description of Poincaré duality for planar graphs.

Exercises follow each section of this book, and there is a dramatic difference between Parts 1 and 2 in this regard. Many of the exercises in Part 1 are of an "easy" or "low-brow" nature in that they ask for verifications of the theorems presented in the text for instance (but to be sure, some of the exercises in Part 1 are of a more interesting sort); this has been done in order to fulfill the needs of several different possible courses based upon this book. In contrast, there are many fewer exercises in Part 2, they are included partly for the convenience of the instructor, are almost all of a substantial nature, and though eclectic, present interesting and/or beautiful aspects of the relevant theory. (It is worth saying that for some optional sections the best exercises are probably just applications and examples of the main results from the text.)

We can have no defense for the choice of topics included as "beautiful" in the optional sections and in Part 2-and for the omission of many other beautiful and important topics as well-other than to ascribe it to our own personal tastes and proclivities and to the constraints of space and time.

As was mentioned above, this book is independent of any knowledge of differential calculus except for certain exercises, and in each of these exercises, we explicitly state that it depends upon calculus. There are furthermore a few exercises in Chapter 10 which depend upon elementary linear algebra, and again this is stated explicitly in each problem.

As to our self-imposed independence from calculus, we might admit that this text has been written in part in reaction to certain trends of calculus reform, which, for better or worse-and simply as a practical matter-have deleted proofs from the traditional syllabus in favor of other algorithmic and electronic aspects. Under the circumstances, we believe it is only sensible that students can profitably learn intellectual rigor and its necessary formalism in studying the foundations of discrete mathematics rather than calculus. (The now-traditional but threatened role of calculus in American college education can apparently be traced to G. H. 
Hardy's lovely and influential book A Course of Pure Mathematics, Cambridge University Press, 1908).

We believe there will be a growing population of students at all levels for courses on discrete mathematics, and we hope this book with its flexible design may be of some pleasant general utility.

Robert C. Penner

Los Angeles, California

July, 1999 